\title{
ESTUDIO DE UN GUANTE DE DATOS EN UN SISTEMA ROBOTIZADO COLABORATIVO PARA CIRUGIA LAPAROSCÓPICA ASISTIDA POR LA MANO
}

\author{
Lidia Santos, José L. González, Eusebio de la Fuente, Juan C. Fraile, Javier P. Turiel. \\ ITAP - Instituto de las Tecnologías Avanzadas de la Producción, Universidad de Valladolid. \\ lidia.santos@uva.es ; \{jossan, efuente,jcfraile, turiel\}@eii.uva.es
}

\begin{abstract}
Resumen
La inclusión de un guante quirúrgico inteligente en una cirugía laparoscópica asistida por la mano dentro de un sistema colaborativo robotizado permitiría enviar información sobre la posición que toma la mano del cirujano durante la intervención. Para ello debe poder definirse los movimientos de la mano de forma univoca. Con dicho guante también se podría definir unas zonas de seguridad en las que dicho sistema robotizado colaborativo no podría trabajar para evitar colisiones con la mano del cirujano. Para ello proponemos la selección de dos tipos de volumen dependiendo de la posición que adopte la mano del cirujano.
\end{abstract}

Palabras Clave: data glove, HALS (Hand Assisted Laparoscopic Surgery), cirugía laparoscópica asistida por la mano, guante de datos.

\section{INTRODUCCIÓN}

La cirugía HALS se caracteriza por la introducción de la mano del cirujano en la cavidad abdominal, recuperando así el sentido del tacto que había perdido en las operaciones laparoscópicas convencionales y dotando a esta cirugía de las ventajas de la cirugía abierta convencional y la cirugía laparoscópica.[1][5][6][7] La cirugía laparoscópica ha ido evolucionando $y$ ha ido incluyendo nuevas herramientas como robots para aportar una mayor estabilidad y precisión en los movimientos de los endoscopios [4,8] hasta la utilización de brazos quirúrgicos como el robot Da-Vinci o robots asistentes semiautónomos [2,3].

Este trabajo forma parte de un proyecto de investigación cuyo objetivo principal es el desarrollo de un sistema robotizado colaborativo para cirugía laparoscópica asistida por la mano. Como parte de este proyecto se desarrollará e integrará en el sistema un guante quirúrgico inteligente y un sistema de visión artificial, además de un sistema de realidad aumentada.
Para ello, en este proyecto, se propone mejorar la funcionalidad del guante del cirujano, incluyendo diferentes sensores para la captación de la posición de la mano pudiendo así realizar operaciones en colaboración con robots quirúrgicos mediante un sistema colaborativo robotizado. Además del guante quirúrgico inteligente, en el proyecto general se incorpora también un sistema de visión que aportará datos relevantes al sistema robotizado colaborativo.

El guante quirúrgico inteligente obtendrá la posición de la mano, que se define como la postura que adoptan en cada momento, es decir al grado de flexión de cada falange y la separación de cada dedo con los contiguos.

A su vez, el sistema de visión determinará la localización de la mano respecto a un eje de referencia determinado.

Con la posición de la mano del cirujano se podrá calcular un volumen aproximado en el que las herramientas del sistema robotizado colaborativo no podrán trabajar por razones de seguridad. Dicho valor junto con la posición y la localización de la mano se envían al sistema robotizado colaborativo para que se puedan utilizar donde corresponda. Dichos datos se utilizarán tanto para evitar colisiones con los minirobots y herramientas laparoscópicas, como para generar un entorno de realidad aumentada con los datos de la cavidad abdominal del paciente y la mano del cirujano.

Para conseguir la integración de los datos, se ha utilizado ROS (Robot Operating System), en su versión Indigo Igloo, bajo entorno Linux (Ubuntu 14.04). ROS es fundamentalmente un sistema cliente/servidor. Consta de una serie de nodos, procesos con una funcionalidad concreta, diseñados de manera modular, que se comunican entre sí a través de topics o services. Los topics son mecanismos de difusión de mensajes mediante el modelo editorsubscriptor en el que uno o varios nodes publican messages en determinados topics al que otros nodes pueden suscribirse. Permite desacoplar producción y consumo de información. Los services son 
mecanismos de comunicación de mensajes de tipo petición/respuesta. Existe un nodo principal, master, al que los nodos acuden para localizarse mutuamente y obtener información de la configuración.

La utilización de ROS permite que los datos estén disponibles a la espera de que diferentes componentes del sistema puedan utilizarlos conjunta o individualmente, como podemos ver en el esquema representado en la Figura 1.

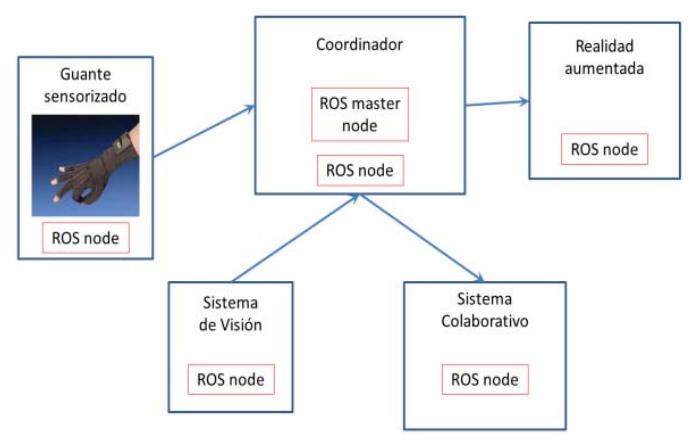

Figura 1: Subsistemas y nodos ROS.

En este artículo nos centraremos en el guante sensorizado y su relación con el resto de componentes.

\section{SELECCIÓN DEL GUANTE 5DT}

Para definir el tipo de sensores que debemos utilizar debemos tener en cuenta el entorno de trabajo, ya que es un espacio muy reducido y no podemos ocupar mucho volumen por lo que debemos minimizar el número de dispositivos a utilizar. Otra variable a considerar es la facilidad que debe aportar el guante quirúrgico inteligente al cirujano a la hora de utilizarlo. Añadir más peso implica peor movilidad en la mano del cirujano lo que dificultaría el desarrollo de la operación. Por ello, la posición de la mano debe quedar definida de forma unívoca utilizando para ello el menor número posible de sensores.

Estas consideraciones no se tienen en cuenta en otros entornos de utilización de los guantes de datos, como pueden ser los videojuegos o el ámbito industrial, ya que en ellos el espacio no suele ser un factor determinante en su utilización al realizarse los movimientos de la mano sin restricciones. Por ello hemos optado por la elección de un guante comercial Data Glove 5DT que se caracteriza por su comodidad y en el uso y el poco volumen ocupado.

El Data Glove 5DT, tanto el de 5 como el de 14 sensores, se caracteriza por la utilización de sensores basados en fibra óptica con una resolución A/D de 12 bits. Viene provisto con un terminal USB 1.1 para realizar la conexión con nuestro PC por lo que se elimina la necesidad de una fuente de alimentación externa. Posee una velocidad de muestreo mínima de $75 \mathrm{~Hz}$.

El guante de 5 sensores posee un sensor por dedo por lo que proporciona un único dato para definir la posición de cada dedo colocado según podemos ver en la Figura 1 (b). Mientras, el guante de 14 sensores posee dos sensores por dedo, uno a nivel de los nudillos (representado por "Sensor 1" en la Figura 1 (a)) y otro a nivel de la primera falange (representado por "Sensor 2" en la Figura 1 (a)), y otro sensor en cada unión de un dedo con el otro dedo (representado por "Unión" en la Figura 1(a)). Por ello el guante de 14 sensores nos proporcionará 2 medidas por dedo para definir la posición de este, además de proporcionarnos la separación o cercanía entre los dedos.

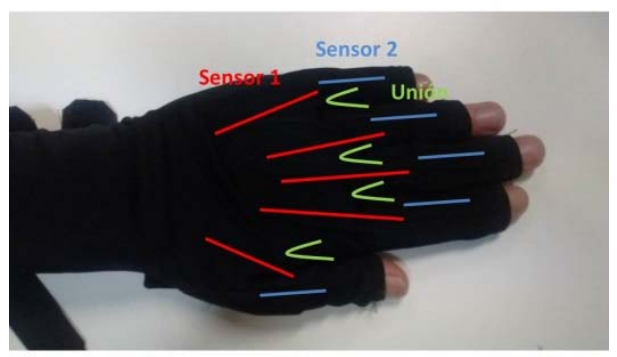

(a)

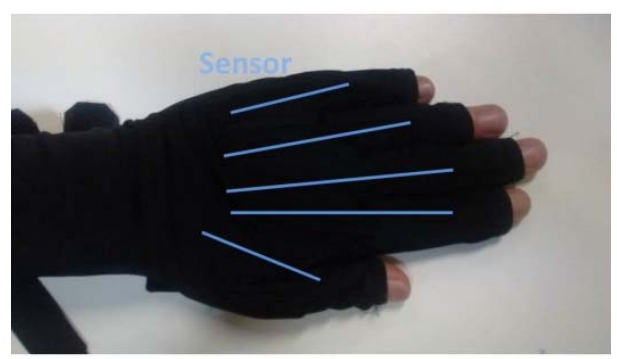

(b)

Figura 2. (a) Posición de los sensores en el guante 5DT de 14 sensores. (b) Posición de los sensores en el guante 5DT de 5 sensores.

El guante se conecta al ordenador mediante conexión USB. El programa de gestión del guante detecta el puerto USB en el que está conectado el guante y comienza la inicialización del guante en la que se recogen los valores del tipo de mano (derecha o izquierda) y el tipo de guante dependiendo del número de sensores que tiene (5 ó 14). Después se procede a la calibración, en la que se recogen los valores máximos y mínimos que detectan los sensores con la mano abierta y la mano cerrada.

El guante, una vez calibrado, pasa a la toma y envío de datos que realizará mediante una función que devuelve 
un vector de números reales con los valores de los 14 sensores. Se recogen los 14 valores de los 5 dedos que serán enviados y se guardarán en un vector llamado datos.

Hemos asignado el nombre "Sensor1" al sensor que mide la flexión de la unión del hueso metacarpiano con su correspondiente falange proximal. En "Sensor1" se sustituirá "Sensor" por el nombre del dedo correspondiente (pulgar, índice, medio, anular o meñique). De igual modo hemos asignado el nombre "Sensor2" al sensor que mide la flexión de la falange proximal con la segunda falange y, al igual que con el caso anterior, hemos sustituido "Sensor" por el nombre del dedo correspondiente. Al sensor que mide la unión entre dos dedos le hemos asignado el nombre "dedo1dedo2" siendo dedo1 y dedo2 los dedos correspondientes a dicha unión. Podemos ver los valores para Anular, en el caso del guante de 5 sensores y Anular1, Anular2 y anularmedio, en el caso del guante de 14 sensores.

Los valores de los sensores se analizan y según las fórmulas (1) y (2), se selecciona el volumen 1 (paralelepípedo) o volumen 2 (semiesfera). Dicho volumen junto con los valores de los diferentes sensores será enviado mediante socket al componente encargado de la comunicación con el sistema robotizado colaborativo. Se utiliza este tipo de comunicación debido a que las librerías de ROS son incompatibles con las proporcionadas para la gestión del guante.

Una vez recibidos dichos datos en el componente encargado de la comunicación con el sistema robotizado colaborativo, éste los publicará en el topic adecuado de manera independiente para que el nodo correspondiente los lea cuando necesite dichos datos. Estas actividades se realizarán con una frecuencia de $10 \mathrm{~Hz}$ por ciclo en el que se actualizarán y publicarán los 14 valores de cada sensor y el volumen elegido.

\subsection{COMPARACIÓN ENTRE GUANTES 5DT DE 5-14 SENSORES.}

En primer lugar, debemos obtener datos de cada dedo ya que tienen movimientos independientes. Teniendo solo esta información no podemos definir de forma unívoca las posiciones que adopta la mano del cirujano ya que los mismos datos podrían interpretarse como varias posiciones. Esto queda reflejado en las siguientes figuras en las que, tomando como ejemplo los datos del dedo anular, el sensor que capta la flexión del dedo anular adopta valores similares, aunque la posición de la mano es diferente.
Teniendo en cuenta los grados de libertad de la mano, consideramos las cuatro posiciones más representativas en las que quedan representados los posibles movimientos de los dedos en las diferentes direcciones posibles: a nivel de la unión del hueso metacarpiano con la falange proximal, la unión de la falange proximal con la falange medial y la separación entre los dedos (interdigital).

Las posiciones $\mathrm{P} 1$ y $\mathrm{P} 2$, con el guante de 5 sensores, generan los mismos datos, por lo que no se podría distinguir cuando adopta una u otra posición. Por ello es necesario la presencia de sensores entre cada dedo para medir su separación

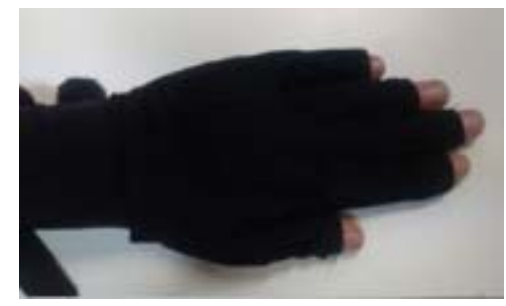

Figura 3. Posición P1.

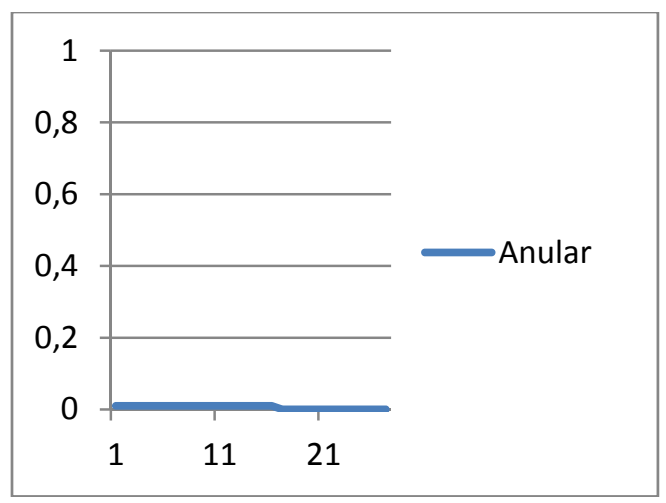

Figura 4. Guante 5DT 5 sensores con posición P1.

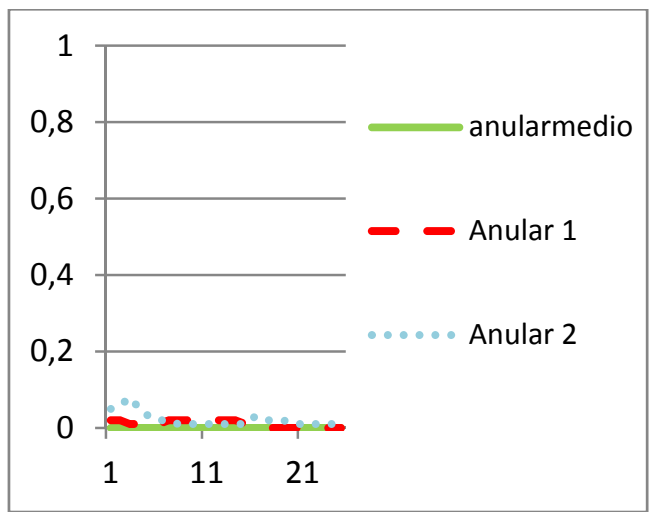

Figura 5. Guante 5DT 14 sensores con posición P1. 


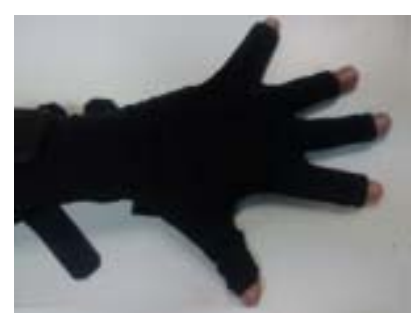

Figura 6. Posición P2.

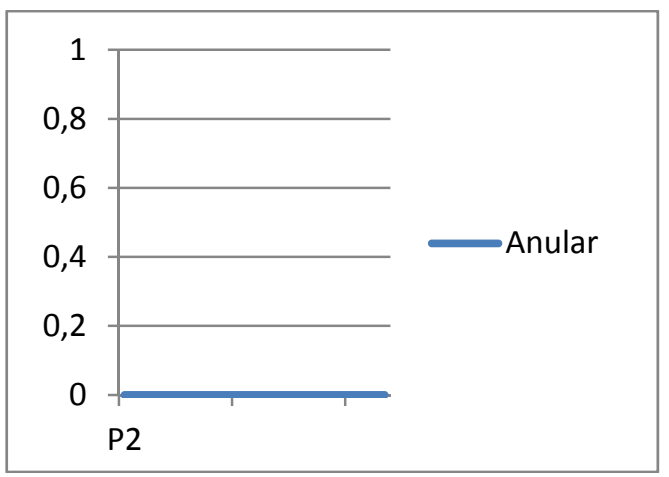

Figura 7. Guante 5DT 5 sensores con posición P2.

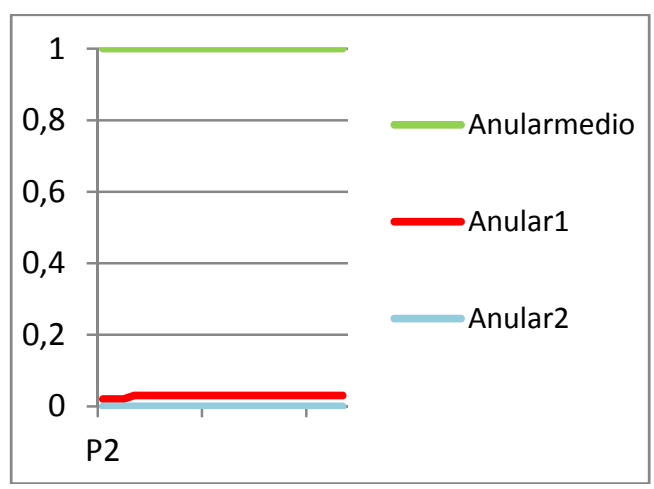

Figura 8. Guante 5DT 14 sensores con posición P2.

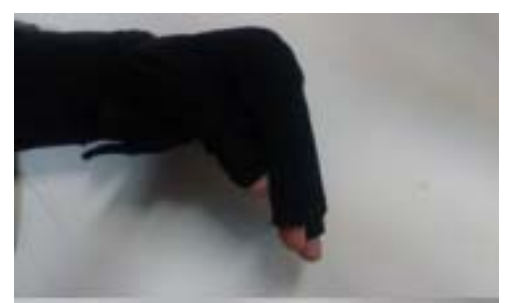

Figura 9. Posición P3.

De igual modo, las posiciones $\mathrm{P} 3$ y $\mathrm{P} 4$ proporcionan los mismos datos por lo que es necesario definir de forma más precisa el movimiento de cada dedo. Adoptando un guante de datos comercial con 14 sensores que miden la flexión de los metacarpianos y la flexión de la primera falange, así como la separación entre los dedos, se consigue parametrizar los datos necesarios de la mano para que el sistema robotizado colaborativo diferencie cada una de las posiciones de la mano.

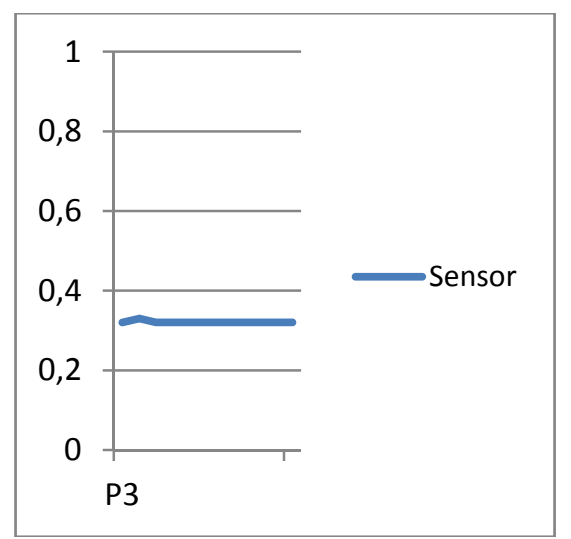

Figura 10. Guante 5DT 5 sensores con posición P3.

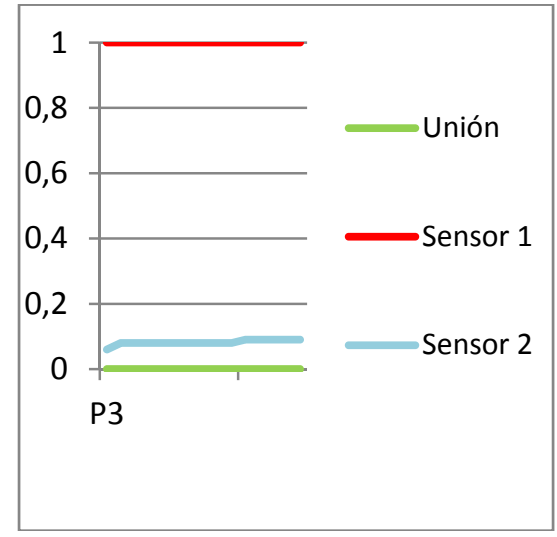

Figura 11. Guante 5DT 14 sensores con posición P3.

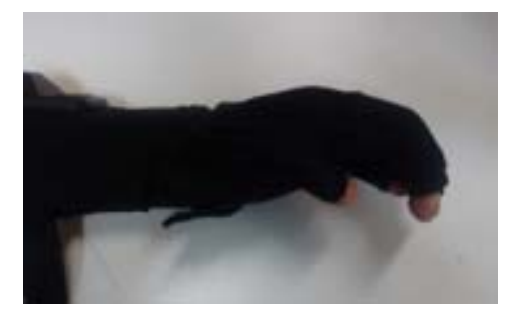

Figura 12. Posición P4.

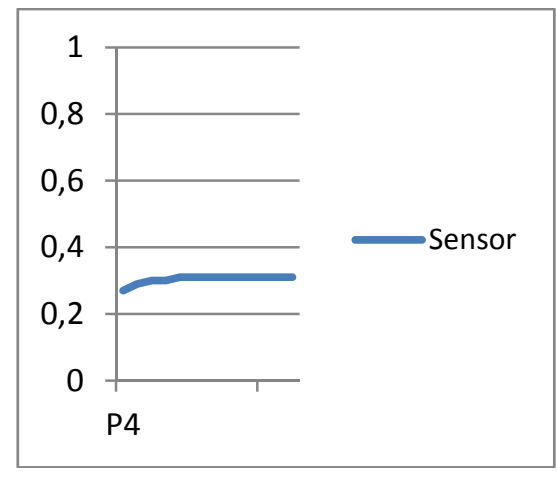

Figura 13. Guante 5DT 5 sensores con posición P4. 


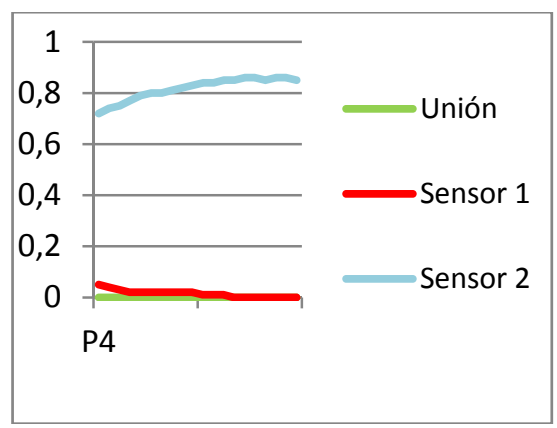

Figura 14. Guante 5DT 14 sensores con posición P4.

La flexión de la segunda falange es dependiente de la flexión de la primera falange por lo que no será necesario enviar este dato para que el sistema colaborativo conozca la posición de la mano.

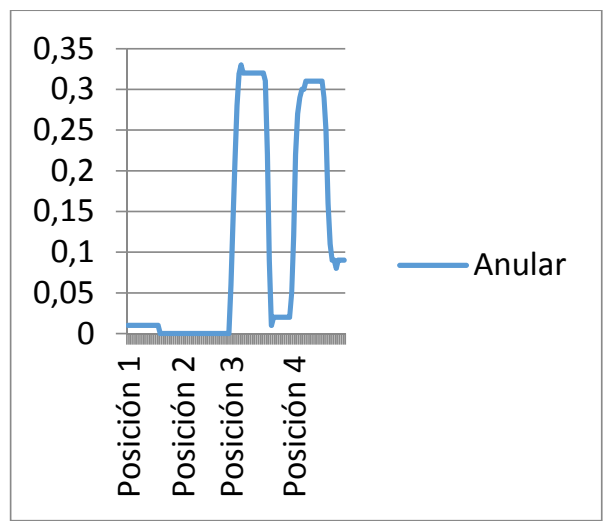

(a)

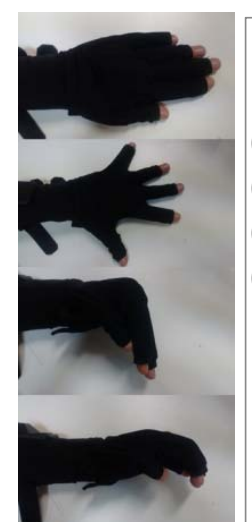

(b)

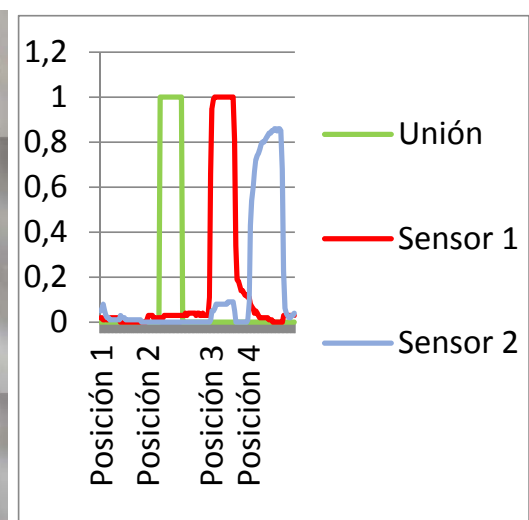

(c)
Figura 15. (a) Valores adoptados por sensor del dedo anular con el guante de 5 sensores al adoptar distintas posiciones. (b) Posiciones 1 a 4 consideradas. (c) Valores adoptados por el sensor del dedo anular con el guante de 14 sensores al adoptar distintas posiciones.

En Figura 15 quedan representados los valores que toman los sensores, tanto del guante de 5 sensores como del guante de 14 sensores, a lo largo del tiempo en el que la mano toma diferentes posiciones (P1, P2, $\mathrm{P} 3, \mathrm{P} 4)$ de forma secuencial según muestra la figura.

\section{VOLUMEN DE TRABAJO}

Para asegurar que la herramienta manejada por el sistema robotizado colaborativo no colisiona con la mano del cirujano se debe definir un volumen de trabajo seguro para dicha mano, en la que la herramienta no podrá operar.

Teniendo en cuenta que la zona de trabajo es la cavidad abdominal en el que no hay mucho espacio, este volumen de trabajo seguro deberá adaptarse a la posición de la mano para que la herramienta tenga más espacio de trabajo. Por la misma razón, la mano del cirujano no dispone de mucho espacio para adoptar diferentes posiciones por lo que la diferencia entre ellas no será muy abrupta y se pueden agrupar varias posiciones dentro de un mismo volumen seguro. Esta opción es más factible que el seguimiento de la posición de la mano en cada momento porque facilita los cálculos y envío de la información al agrupar diferentes movimientos dentro de la misma zona segura de trabajo.

Se ha considerado que los movimientos que realiza el cirujano con los dedos índice, medio, anular y meñique suelen realizarse con el movimiento del dedo pulgar. También se considera que las flexiones de los dedos hacia atrás son irrelevantes tanto para definir la posición de la mano como para definir el volumen de seguridad debido a que al introducir la mano el cirujano a través del puerto único para realizar una operación de tipo HALS (Hand Assited Laparoscopic Surgery), queda situada de tal manera que las herramientas nunca van a estar situadas en la parte posterior de la mano. Por todo ello, en el desarrollo de este trabajo, se han reducido a dos los volúmenes de trabajo seguro definidos, el primero mediante un paralelepípedo y el segundo mediante una semiesfera.

Las medidas del paralelepípedo vendrán predefinidas por la fisionomía de la mano del cirujano por lo que para la longitud del paralelepípedo se tiene en cuenta la distancia de la unión del hueso metacarpiano con los huesos carpianos a la punta del dedo medio; para la anchura se considera la punta del dedo meñique con la punta del dedo pulgar teniendo en cuenta que para tomar dicha medida deberá estar la mano extendida como se indica en la Posición P2. Dichas medidas serán proporcionadas por el sistema de visión. La altura (a) también estará prefijada ya que las mano suelen tener similares medidas respecto a esa distancia. Tanto a la longitud del paralelepípedo como a la anchura se le suma una distancia de seguridad. 


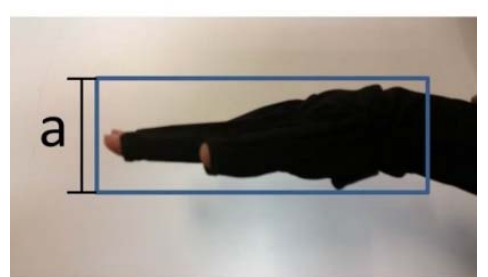

(a)

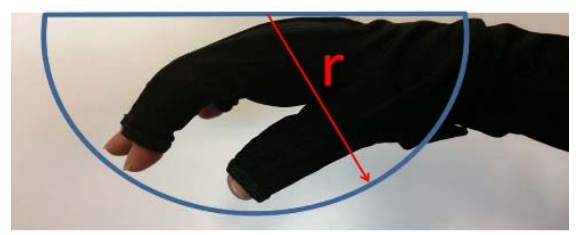

(b)

Figura 16. (a) Volumen con forma de paralelepípedo definido en torno a la mano. (b) Volumen con forma de semiesfera definido en torno a la mano.

Las medidas de la semiesfera también vendrán dadas por las características de la fisionomía de la mano del cirujano siendo el radio de la semiesfera igual a la distancia entre la unión del hueso metacarpiano del dedo medio y la punta del dedo pulgar cuando la mano adopta la Posición P2. El centro O de la semiesfera vendría dado por la unión entre el hueso metacarpiano del dedo medio con la falange proximal de dicho dedo. Ambas medidas serán proporcionadas por el sistema de visión.

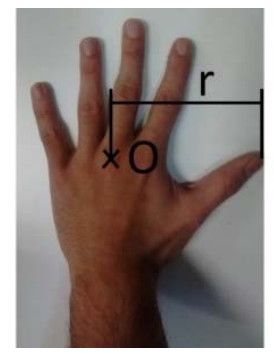

Figura 17. Definición del radio r y el centro O.

Al adoptar la mano una postura cualquiera, los sensores, tanto a la altura del metacarpo como a la altura de la falange proximal, captarán los ángulos de flexión de cada dedo y proporcionarán un valor de 12 bits para definirlos. Cuando los ángulos de flexión indiquen que los dedos del cirujano están fuera del volumen de seguridad definido por el paralelepípedo, teniendo en cuenta siempre el caso más restrictivo, se adoptará el volumen de seguridad definido por la semiesfera, con lo que la mano del cirujano volverá a estar dentro de un volumen de seguridad evitando así posibles colisiones con las herramientas manejadas por el sistema robotizado colaborativo. Al volver a adoptar la mano cualquier posición que pueda definirse dentro del volumen del paralelepípedo, se volverá a adoptar el volumen de seguridad representado por un paralelepípedo, ya que con este volumen se proporciona un mayor espacio de trabajo a las herramientas al ser el volumen del paralelepípedo menor que el de la semiesfera.

Para seleccionar una opción u otra se tiene en cuenta el ángulo de flexión que proporcionan los sensores situados en los metacarpos y en la falange proximal. Primero se mide el ángulo de flexión del metacarpo (ángulo $\alpha$ ) o de la primera falange (ángulo $\beta$ ) de cada dedo. Se considera ' $y$ ' a la distancia entre la unión de la falange medial y la falange proximal y la punta del dedo que se va a flexionar, $y$ ' $z$ ' la distancia entre la punta del dedo que se va a flexionar y la unión entre su falange proximal y su respectivo hueso metacarpiano. Dichas medidas serán proporcionadas offline por el sistema de visión y son parámetros propios de la mano del cirujano.

Para las condiciones definidas por la ecuación (1) se considera la opción de la semiesfera y para las condiciones definidas en (2) se considera la opción del paralelepípedo, quedando así siempre los dedos del cirujano dentro de un volumen de trabajo seguro.

$$
\begin{aligned}
& {[z \cdot \operatorname{sen}(\alpha)+y \cdot \operatorname{sen}(\beta) \geq a]} \\
& {[z \cdot \operatorname{sen}(\alpha)+y \cdot \operatorname{sen}(\beta)<a]}
\end{aligned}
$$

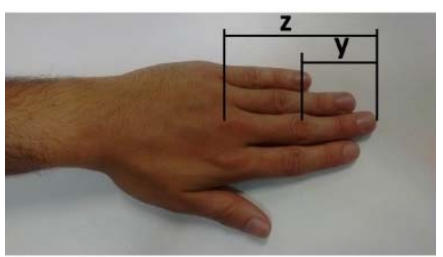

(a)

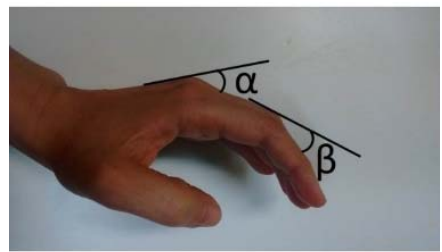

(b)

Figura 18. (a) Definición de las medidas y, z. (b) Definición los ángulos $\alpha$ y $\beta$.

La comunicación de los elementos del sistema robotizado colaborativo se realiza mediante ROS (Robot Operating System) de tal manera que el sistema desarrollado en torno al guante de datos ejerce la función de editor tanto de cada una de las medidas 
captadas por los 14 sensores como de la opción de volumen segura adecuada en cada momento.

Cada elemento suscrito a estos datos dentro del sistema robotizado, podrá recibir dichos parámetros y los podrá procesar para identificar la posición de la mano del cirujano, pudiendo así identificar la fase de la operación en la que se encuentra, y para descifrar el volumen en el que puede operar de forma segura.

Para identificar la localización de la mano dentro de la cavidad abdominal se utilizará un sistema de visión artificial cuyos datos también serán comunicados mediante ROS. Los datos del sistema de visión se procesarán junto con los datos del guante para definir la localización de la mano, ya que los datos del guante sólo nos proporcionan la posición en la que se encuentra la mano y el volumen de seguridad alrededor de ella en el que no pueden trabajar las herramientas dirigidas por el sistema robotizado colaborativo, pero no nos proporciona dónde se encuentra la mano respecto a ninguna referencia.

\section{CONCLUSIONES}

Para determinar las dimensiones del paralelepípedo y la semiesfera que definirán el espacio de trabajo donde no podrá operar el robot, necesitaremos saber las dimensiones de la mano del cirujano, por lo que se obtendrán por visión artificial mientras que las flexiones de los metacarpos y primeras falanges de cada dedo se obtendrán por el guante de datos ya que no podrían obtenerse por visión artificial debido a las dificultades en el campo de visión dentro de la cavidad abdominal.

La comunicación de los datos entre el sistema de visión, el guante de datos y el sistema robotizado colaborativo se lleva a cabo mediante ROS ya que proporciona el soporte adecuado mediante una comunicación tipo editor-suscriptor.

Con cinco sensores no queda definida la posición de la mano de forma unívoca por lo que necesitaremos, al menos 14 sensores.

\section{Agradecimientos}

Este trabajo ha sido financiado por el Ministerio de Economía y Competitividad, a través del proyecto: "Sensorized HALS. Entorno sensorizado para cirugía laparoscópica asistida por la mano". Subproyecto dentro del proyecto coordinado "Sistema robotizado colaborativo para cirugía laparoscópica asistida por la mano". DPI2013-47196-C3-3-R.

\section{Referencias}

[1] Aalbers G. J., Doeksen a, Van Berge M. I. Henegouwen, and Bemelman W. a, (2010) "Hand-assisted laparoscopic versus open approach in colorectal surgery: a systematic review", Colorectal Dis., vol. 12, no. 4, pp. 287295.

[2] Bauzano, E., (2013) “A minimally invasive surgery robotic assistant for HALS-SILS techniques", Computer Methods and Programs in Biomedicine, 112:2, November 2013, pp. 272283.

[3] Bauzano, E., Garcia-Morales, I., del Saz-Orozco, P., Fraile, J.C., Muñoz, V.F., (2014) "Robot collaborative assistance for suture procedures via minimally invasive surgery", Advances in Intelligent Systems and Computing, 252, 2014, pp. 255-269.

[4] Gorey, T.F., Tierney, S., Buckley, D., O'Riordain, M., Fitzpatrick, J.M., (1996) "Video-assisted Nissen's fundoplication using a hand-access port", Minimally Invasive Therapy and Allied Technologies, 5 (4), pp. 364-366.

[5] Jayne D.G., Thorpe H.C., Copeland J., Quirke P., Brown J.M., and Guillou P. J., (2010) "Five-year follow-up of the Medical Research Council CLASICC trial of laparoscopically assisted versus open surgery for colorectal cancer," Br. J. Surg., vol. 97, no. 11, pp. 1638-1645.

[6] Nakajima K., Lee S. W., Cocilovo C., Foglia C., Sonoda T., and Milsom J. W., (2004) "Laparoscopic total colectomy: Hand-assisted vs standard technique," Surg. Endosc. Other Interv. Tech., vol. 18, no. 4, pp. 582-586.

[7] Swanson T. W., Meneghetti A. T., Sampath S., Connors J. M., and. Panton O. N. M,(2011) "Hand-assisted laparoscopic splenectomy versus open splenectomy for massive splenomegaly: 20-Year experience at a Canadian centre," Can. J. Surg., vol. 54, no. 3, pp. 189-193.

[8] Taylor, R.H., Funda, J., Eldridge, B., Gomory, S., Gruben, K., LaRose, D., Talamini, M., Kavoussi, L., Anderson, J., (1995) “A telerobotic assistant for laparoscopic surgery", IEEE Engineering in Medicine and Biology Magazine, $14: 3,1995$, pp. 279-288. 